Research Article

Alberto Carpinteri, Giuseppe Lacidogna*, and Domenico Scaramozzino

\title{
Geometrically nonlinear behavior of lattice domes coupled with local Eulerian instability
}

https://doi.org/10.1515/cls-2020-0020

Received Jun 18, 2020; accepted Sep 30, 2020

\begin{abstract}
Structural analysis is an intricate subject when nonlinearities occur. They make the structural behavior complex and may have important consequences in the design choice as well. Especially for lattice domes, as snapthrough phenomena and local Eulerian instabilities generally affect the structural response, linear analysis is not enough. In this paper, a semi-analytical formulation is used in order to study the geometrically nonlinear behavior of lattice domes subject to vertical loads. The formulation is derived from the equilibrium equations written in the deformed configuration, considering large displacements and taking also into account local buckling conditions. The resulted system of equations, being strongly nonlinear, has been solved by means of a numerical procedure, based on a mixed load-displacement control scheme, leading to the evaluation of the complete equilibrium path. The influence of geometrical parameters on the critical load multiplier and shape of the load-displacement curve is also discussed. In particular, a complex equilibrium path for a sixteen-member five-node lattice structure is analyzed, which is characterized by several branches which can generate 'snapping' conditions.
\end{abstract}

Keywords: geometrically nonlinear analysis, mechanical instability, snap-through, critical load multiplier, local buckling, equilibrium path

\section{Introduction}

In the field of Structural Engineering, one of the main issues when dealing with thin, slender or long-span struc-

^Corresponding Author: Giuseppe Lacidogna: Department of Structural, Geotechnical and Building Engineering, Politecnico di Torino, Corso Duca degli Abruzzi 24 - 10129 Torino, Italy; giuseppe.lacidogna@polito.it

Alberto Carpinteri, Domenico Scaramozzino: Department of Structural, Geotechnical and Building Engineering, Politecnico di Torino, Corso Duca degli Abruzzi 24 - 10129 Torino, Italy tures is represented by the occurrence of catastrophic failures which are associated with instability phenomena, e.g. the sudden collapse of the single-layer lattice dome in Bucharest (Romania) which occurred in 1963 as a result of local snap-through due to an unexpected snow accumulation on a small area [1]. Predicting these kinds of failure is generally challenging, since there exist several factors which play a key role in determining the critical load factor as well as the collapse mode.

Plenty of research has been conducted on the subject of structural instabilities especially in the twentieth century. For example, in the same year in which the Bucharest dome collapsed (1963), Thompson published an article in which the basic principles of 'snapping' and 'buckling' instabilities were examined and defined from a mathematical perspective [2]. Several years before, the subject of buckling instability in thin and slender elements was deeply investigated by Von Karman via a theoretical approach [3-5]. Nonlinear load-displacement curves, highlighting potential snap-through instabilities, were found when analyzing spherical shells under external pressure [3] and curved bars under concentrated loads [4]. The same theoretical framework was applied by Von Karman and Tsien [5] and Michielsen [6] to investigate the buckling of thin cylinders subjected to axial compression: in this case, the buckling collapse was associated to sharp cusp points in the stress-strain equilibrium curve, underlying potential snap-back instabilities.

It is worth noting that these different kinds of instabilities (i.e. local buckling, snap-through, snap-back) do not depend only on the considered structural system (e.g. axially-loaded cylinders, spherical shells under pressure, etc.), but all of them can occur in the same structural element when changing its geometrical or material properties. For example, in his work [7], Bushnell showed how the load-deflection curves of a spherical cap clamped at the edges, under external pressure, vary when changing a slenderness parameter $\lambda$, defined as follows:

$$
\lambda=2\left[3\left(1-v^{2}\right)\right]^{\frac{1}{4}}\left(\frac{H}{h}\right)^{\frac{1}{2}},
$$

being $v$ the Poisson's ratio of the material, $H$ the total rise of the spherical cap above the plane in which the edges lie 


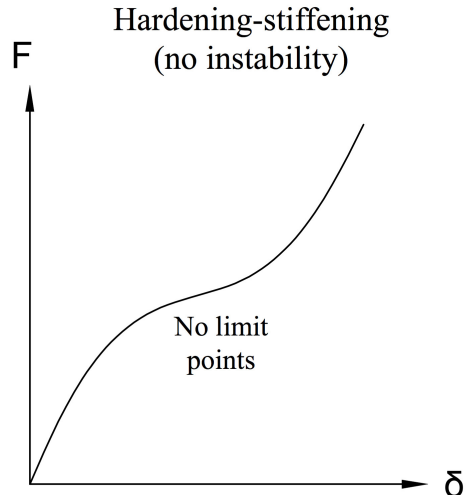

(a)

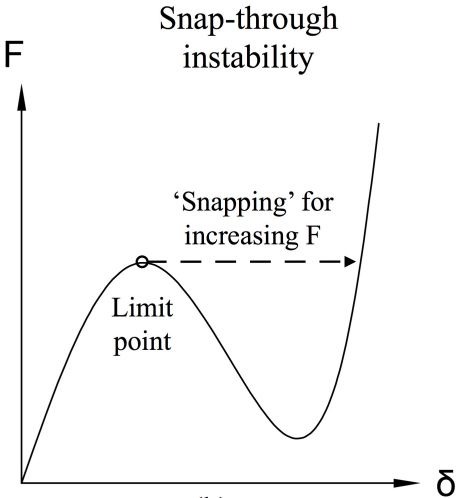

(b)

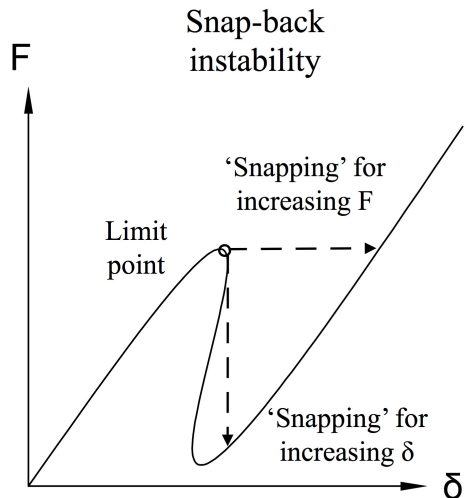

(c)

Figure 1: Equilibrium curves exhibiting different stability phenomena: (a) hardening-stiffening behavior (no instability); (b) snap-through instability; (c) snap-back instability

and $h$ the shell thickness. It was found that, by increasing $\lambda$ (i.e. by decreasing the shallowness of the cap), the shape of the equilibrium path changed considerably. Hardeningstiffening behavior was observed for low $\lambda$ values $(\lambda<3.5)$, snap-through-like curves were obtained for medium $\lambda$ values $(3.5<\lambda<7)$, whereas cusp limit points with snapback branches were found for higher values $(\lambda>7)$ [7]. This was mainly due to the fact that, by decreasing the shallowness, the spherical cap passed from a purely flexural regime to a mixed membrane-flexural condition, and this strongly modifies both the equilibrium path and the instability collapse mode.

Hence, it is evident that geometric properties can affect the structural behavior in a way that they modify the shape of the load-deflection curve and have a crucial influence on the structural stability. Summarizing, we may assume to deal with the three general cases for the equilibrium curves when considering large displacements, as shown in Figure 1, i.e. no instability phenomena, if no limit points are found in the load-deflection path (Figure 1a), snap-through-like instabilities, if some limit points can produce a 'snapping' of the structure for increasing loads (Figure 1b), and snap-back-like instabilities, if the limit points can trigger a 'snapping' for increasing loads and displacements (Figure 1c). These equilibrium curves may also be affected by bifurcation points, which are generally associated to local buckling conditions. It is interesting to observe that snap-back instabilities were also obtained when considering critical failure phenomena in materials, e.g. within unstable fracture processes in brittle and quasibrittle materials [8], as well as during friction contact loss between rough surfaces (stick-slip phenomenon) [9]. In a sense, snap-back phenomena may be considered as the unified concept of catastrophic collapses, which is common to structures and materials.

As briefly mentioned above with respect to the collapse of the Bucharest dome, the problem of structural stability is pivotal when dealing with lattice space structures [10-13]. These have been ones of the most exploited structural systems for the realization of long-span roofs since the second half of the twentieth century. Moreover, starting from the last few decades, they have experienced a further significant growth among other structural solutions. Remarkable examples around the world are the Frei Otto's Multihalle (Mannheim), the Arata Isozaki's Palau Sant Jordi (Barcelona), the Frank O. Ghery's DZ Bank Building (Berlin), the Foster's Queen Elizabeth II Great Court (London), etc. Two main factors can be evidenced which induced the growing success of lattice domes. On one side, being composed by the assembly of bars generating different geometrical patterns (triangular, hexagonal, etc.), these structures can lead to remarkable architectural effects, allowing engineers and architects to realize impressive complex-shaped long-span roofs. On the other hand, light and slender structures can be obtained, especially when optimization processes are conducted aimed at minimizing the amount of structural weight, while complying with safety standards as well.

Dealing with this kind of structures, material resistance is not the only driving parameter affecting the maximum loading capacity, as this can be strongly affected by instability phenomena, such as local Eulerian buckling and global instabilities [10]. Furthermore, coupled instability phenomena might also occur and the interaction between different collapse modes may be crucial in determining the bearing capacity of the structure [14-16]. Therefore, from a design point of view, starting from a defined load 
scheme, the search for the critical load factor is needed, which can be associated to single member failures as well as to the collapse of entire dome portions, and geometric nonlinear analysis must be performed in order to trace the equilibrium path $[10,17,18]$.

In addition to buckling failures which affect single bar members or dome portions, the most important stability problem occurring in this kind of structure is usually associated to the snap-through phenomenon (Figure 1b). It arises when the structure reaches its maximum loading capacity and, if the load still increases a little, the construction suddenly changes its shape in order to reach a new equilibrium configuration $[17,18]$. In most cases, this conformational change is incompatible with the structure serviceability and, as this always occurs with high dynamical effects, it can cause the sudden collapse of the structure $[1,19]$. Finally, it is important to note that in real structures the members are characterized by geometrical and mechanical imperfections. These can reduce the critical load even more, interacting both with the local Eulerian instability and the snap-through phenomenon $[10,20]$.

In this paper, a semi-analytical formulation has been used for the evaluation of the geometrically nonlinear behavior of lattice domes under vertical loads. It is based on the equilibrium equations written in the deformed configuration of the structure, taking also into account the possible occurrence of local buckling condition. Due to the fact that the resulting system is composed by nonlinear equations, a numerical method has been used in order to solve the structural problem. For a well-defined load scheme, the semi-analytical formulation allowed to trace the entire nonlinear equilibrium path and to identify the critical load factors and the correspondent instability modes of different lattice structures.

\section{Methodology}

As mentioned in the Introduction, in this paper a semianalytical formulation is used in order to perform the geometric nonlinear analysis of lattice domes, taking also into account local Eulerian instabilities. A generic lattice dome is considered in a three-dimensional reference system, made up of $N$ free nodes and $V$ external constrained nodes. At this stage and in favor of structural safety as regards instability phenomena, both internal and external constraints are assumed to be perfect hinges without friction. Moreover, the eccentricity between the members converging into the nodes are neglected, as well as the imperfections of the bars, which will be addressed in future re- search efforts; therefore, the bars are subject only to axial forces. Finally, the load scheme is assumed as a combination of vertical forces acting on free nodes, which generate vertical node displacements.

The vertical forces acting on the $N$ free nodes being grouped into the $N$-size vector $\mathbf{F}$ and the corresponding vertical node displacements into the $N$-size vector $\boldsymbol{\delta}$, the relation between $\mathbf{F}$ and $\boldsymbol{\delta}$ can be formalized into the following analytical form:

$$
\boldsymbol{F}=\boldsymbol{f}(\boldsymbol{\delta}),
$$

where $\boldsymbol{f}$ is the (nonlinear) system linking the force vector to the displacement vector. Eq. (2) can be expanded as follows, highlighting the terms referring to each node of the structure:

$$
\left\{\begin{array}{c}
F_{1} \\
\ldots \\
F_{i} \\
\ldots \\
F_{N}
\end{array}\right\}=\left\{\begin{array}{c}
f_{1}(\boldsymbol{\delta}) \\
\ldots \\
f_{i}(\boldsymbol{\delta}) \\
\ldots \\
f_{N}(\boldsymbol{\delta})
\end{array}\right\}=\left\{\begin{array}{c}
f_{1}\left(\delta_{1}, \ldots \delta_{N}\right) \\
\ldots \\
f_{i}\left(\delta_{1}, \ldots \delta_{N}\right) \\
\ldots \\
f_{N}\left(\delta_{1}, \ldots \delta_{N}\right)
\end{array}\right\} .
$$

In Eq. (3) the nonlinear system which characterizes the structural problem is directly represented: it is a system of $N$ nonlinear scalar equations $f_{1}, \ldots f_{N}$ depending on the $N$ unknowns $\delta_{1}, \ldots \delta_{N}$. The nonlinear functions contained in Eq. (3) can be analytically written down if a general displacement vector $\boldsymbol{\delta}$ is applied to the structure, and the equilibrium equations are formulated in each node within the deformed configuration.

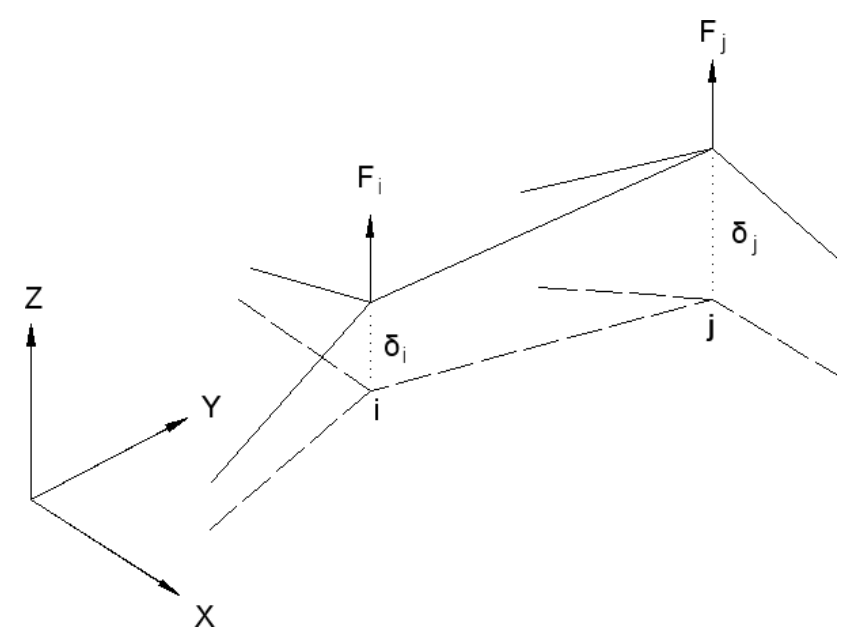

Figure 2: Reference geometrical scheme for the semi-analytical formulation (unloaded structure is shown by dotted lines, deformed structure by continuous lines)

The vector $\boldsymbol{\delta}$ being applied to the structure, the $i^{\text {th }}$ and $j^{\text {th }}$ nodes undergo vertical displacements $\delta_{i}$ and $\delta_{j}$ respectively (Figure 2). The bar connecting the $i^{\text {th }}$ and $j^{\text {th }}$ nodes, 
which in the initial configuration was characterized by a length $L_{i j}$ equal to:

$$
L_{i j}=\sqrt{\left(x_{i}-x_{j}\right)^{2}+\left(y_{i}-y_{j}\right)^{2}+\left(z_{i}-z_{j}\right)^{2}},
$$

in the deformed configuration is characterized by a different length $L^{\prime}{ }_{i j}$, which is equal to:

$$
L^{\prime}{ }_{i j}=\sqrt{\left(x_{i}-x_{j}\right)^{2}+\left(y_{i}-y_{j}\right)^{2}+\left(\left(z_{i}+\delta_{i}\right)-\left(z_{j}+\delta_{j}\right)\right)^{2}} .
$$

Thus, the axial deformation of the bar connecting the $i^{\text {th }}$ and $j^{\text {th }}$ nodes can be easily calculated evaluating the relative engineering strain, as follows:

$$
\begin{aligned}
\epsilon_{i j} & =\frac{L_{i j}^{\prime}-L_{i j}}{L_{i j}} \\
& =\sqrt{\frac{\left(x_{i}-x_{j}\right)^{2}+\left(y_{i}-y_{j}\right)^{2}+\left(\left(z_{i}+\delta_{i}\right)-\left(z_{j}+\delta_{j}\right)\right)^{2}}{\left(x_{i}-x_{j}\right)^{2}+\left(y_{i}-y_{j}\right)^{2}+\left(z_{i}-z_{j}\right)^{2}}}-1 .
\end{aligned}
$$

In this study, only geometrical nonlinearities are addressed, therefore an elastic constitutive law for the material is assumed. Indicating by $E_{i j}$ and $A_{i j}$ the material Young's Modulus and the bar cross-sectional area respectively, the axial force $N_{i j}$ can be evaluated as follows:

$$
\begin{aligned}
& N_{i j}=E_{i j} A_{i j} \epsilon_{i j}=E_{i j} A_{i j} \\
& \left(\sqrt{\frac{\left(x_{i}-x_{j}\right)^{2}+\left(y_{i}-y_{j}\right)^{2}+\left(\left(z_{i}+\delta_{i}\right)-\left(z_{j}+\delta_{j}\right)\right)^{2}}{\left(x_{i}-x_{j}\right)^{2}+\left(y_{i}-y_{j}\right)^{2}+\left(z_{i}-z_{j}\right)^{2}}}-1\right) .
\end{aligned}
$$

Referring to Figure 2, the equilibrium equation to the vertical translation can be then formulated in the $i^{\text {th }}$ node where the external applied force $F_{i}$ is balanced by the axial forces in the bars converging into the node. Decomposing the axial forces along the vertical direction, one obtains the following equation:

$$
F_{i}=\sum_{k} N_{i k} \frac{\left(z_{i}+\delta_{i}\right)-\left(z_{k}+\delta_{k}\right)}{L^{\prime}{ }_{i k}},
$$

where the index $k$ refers to the $k^{\text {th }}$ node, which is linked to the $i^{\text {th }}$ node. Substituting Eq. (7) into Eq. (8), the analytical form of the $i^{\text {th }}$ nonlinear function $f_{i}\left(\delta_{1}, \ldots \delta_{N}\right)$ reported in Eq. (3) can be finally obtained, as follows:

$$
\begin{aligned}
& F_{i}=\sum_{k} E_{i k} A_{i k} \\
& \left(\sqrt{\frac{\left(x_{i}-x_{j}\right)^{2}+\left(y_{i}-y_{j}\right)^{2}+\left(\left(z_{i}+\delta_{i}\right)-\left(z_{j}+\delta_{j}\right)\right)^{2}}{\left(x_{i}-x_{j}\right)^{2}+\left(y_{i}-y_{j}\right)^{2}+\left(z_{i}-z_{j}\right)^{2}}}-1\right) \\
& \left(\frac{\left(z_{i}+\delta_{i}\right)-\left(z_{k}+\delta_{k}\right)}{L^{\prime}{ }_{i k}}\right) .
\end{aligned}
$$

The previous equation is valid only if the axial force in the generic bar connecting the $i^{\text {th }}$ and $k^{\text {th }}$ nodes, expressed by Eq. (7), is lower than the Euler critic load. This is defined by the following expression:

$$
N_{c r, i j}=\pi^{2} \frac{E_{i j} I_{i j}}{L_{i j}{ }^{2}},
$$

$I_{i j}$ being the minimum moment of inertia of the bar section. If the axial force expressed by Eq. (7) exceeds the Euler critic load defined by Eq. (10), the bar buckles and cannot sustain further axial load increases. Therefore, the complete analytical formulation of the nonlinear function $f_{i}\left(\delta_{1}, \ldots \delta_{N}\right)$, which takes also into account the buckling conditions of the single members, is represented by the following equation:

$$
\begin{aligned}
F_{i} & =\sum_{k, n b} E_{i k} A_{i k}\left(\frac{L^{\prime}{ }_{i k}}{L_{i k}}-1\right)\left(\frac{\left(z_{i}+\delta_{i}\right)-\left(z_{k}+\delta_{k}\right)}{L^{\prime}{ }_{i k}}\right) \\
& +\sum_{k, b} \pi^{2} \frac{E_{i k} I_{i k}}{L_{i k}{ }^{2}}\left(\frac{\left(z_{i}+\delta_{i}\right)-\left(z_{k}+\delta_{k}\right)}{L^{\prime}{ }_{i k}}\right),
\end{aligned}
$$

where the first summation involves not buckled bars, while the second one refers to buckled members.

In order to solve the structural problem reported in Eq. (1), the system being composed by nonlinear equations, a numerical procedure implemented into the MATLAB ${ }^{\circledR}$ environment has been used. The analysis starts from the unloaded state, in which both the force and displacement vectors are null. Then, the load scheme is defined through an initial force vector $\boldsymbol{F}_{\mathbf{0}}$, and the corresponding structure displacements are obtained from Eq. (2). Since the nonlinear system reported in Eq. (2) is not directly invertible, the displacements corresponding to $\boldsymbol{F}_{\mathbf{0}}$ are obtained by using MATLAB ${ }^{\circledR}$ tools which find the roots of analytical functions starting from a close input solution. Starting from the input solution which is set as the null displacement vector (i.e. $\boldsymbol{\delta}=\mathbf{0}$ ), the displacement vector $\boldsymbol{\delta}_{\mathbf{0}}$ which satisfies the following system can then be obtained:

$$
\boldsymbol{f}\left(\boldsymbol{\delta}_{\mathbf{0}}\right)-\boldsymbol{F}_{\mathbf{0}}=\mathbf{0} .
$$

Subsequently, the load factor $\alpha$ is introduced which multiplies the initial load vector $\boldsymbol{F}_{\mathbf{0}}$. At each step, the load factor is increased and the starting input solution is set as the previously obtained displacement vector. The subsequent equation is then solved using the above mentioned MATLAB ${ }^{\circledR}$ functions to follow the nonlinear equilibrium path of the structure:

$$
\boldsymbol{f}(\boldsymbol{\delta}(\alpha))-\alpha \boldsymbol{F}_{\mathbf{0}}=\mathbf{0} .
$$

As can be inferred, the problem has been solved by adopting a load-control scheme, i.e. by increasing the load 
vector via the factor $\alpha$ and solving at each step the nonlinear system reported in Eq. (13). However, since the structural problem defined by Eq. (13) contains nonlinear functions which may exhibit limit points, i.e. relative maxima and minima, the numerical procedure has been implemented in a way that, when a local maximum or minimum is reached, an alert flag is activated. In that case, a local displacement-control scheme becomes active just in order to overcome the limit point, i.e. looking for a solution of Eq. (13) beyond the detected limit point. After passing the limit point, the numerical procedure takes back onto the load-control scheme, taking into account the fact that a local maximum or minimum was just reached. If the load factor was previously increasing and the alert flag becomes active, that means that a local maximum has been reached and the load factor is subsequently made decreasing after passing the limit point; vice versa, if the load factor was previously decreasing and the alert flag becomes active, that means that a local minimum has been reached and the load factor is subsequently made increasing. In this way, the complete equilibrium path could generally be followed. It is worth mentioning that the problem of tracing nonlinear equilibrium paths and passing limit points has been an important issue for long time, and several numerical methodologies have been proposed [21-25]. Today, the most efficient methods are already implemented within various commercial software.

\section{Results and discussion}

Based on the semi-analytical formulation described in Section 2, three examples of lattice dome structures are presented here.

\subsection{Eight-member lattice structure with one free node}

The first example refers to a structure made up of eight constrained nodes and one top free node, as shown in Figure 3. The eight bars are hinged to the ground and to the top node, and the structure is subject to a downward force applied on the top node, whose initial value is equal to $20 \mathrm{kN}$ (corresponding to $\alpha=1$ ).

The Young's Modulus and the cross-sectional area are set equal for the eight members and their values are reported in Table 1. In order to investigate the influence of Eulerian instabilities on the geometrically nonlinear behavior of the structure, three different moments of inertia have

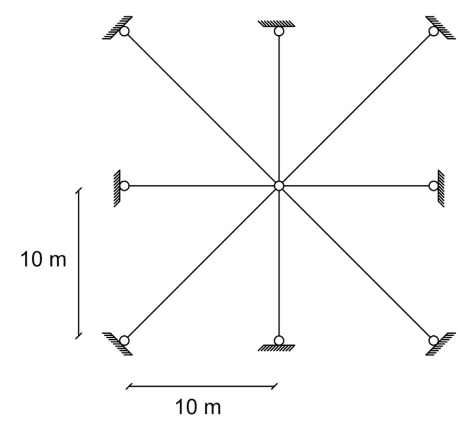

(a)

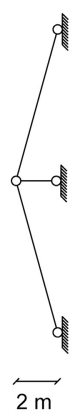

(b)
Figure 3: Geometry of the eight-member lattice structure: (a) top view; (b) lateral view

been assigned to the bars, so that three slenderness values are considered for these members (Table 1). The slenderness is defined as:

$$
\lambda=\frac{L}{\sqrt{\frac{I}{A}}},
$$

$L$ being the bar length, $I$ and $A$ its minimum moment of inertia and its cross-sectional area, respectively.

Table 1: Eight-member lattice structure properties

\begin{tabular}{cccc}
\hline Case & 1 & 2 & 3 \\
\hline $\mathrm{E}[\mathrm{GPa}]$ & 30 & 30 & 30 \\
$\mathrm{~A}\left[\mathrm{~cm}^{2}\right]$ & 100 & 100 & 100 \\
$\mathrm{I}_{\min }\left[\mathrm{cm}^{4}\right]$ & $3.2 \cdot 10^{5}$ & $8 \cdot 10^{4}$ & $4 \cdot 10^{4}$ \\
$\lambda_{\max }[-]$ & 25.2 & 50.5 & 71.4 \\
$\alpha_{\text {crit }}[-]$ & 121 & 96 & 55 \\
\hline
\end{tabular}

In Figure 4 the equilibrium paths of the structure are reported for the three cases. As can be noted, the higher the bar slenderness, the more important the Eulerian buckling phenomenon in determining the critical load factor. In particular, in Case $1\left(\lambda_{\max }=25.2\right)$ the critical load factor is equal to 121 and no Eulerian instabilities occur, whereas in Case 2 and 3 the critical load factor is equal to 96 and 55, respectively, and the snap-through instability is activated by the Eulerian buckling, as can be observed from the presence of bifurcation points within the load-deflection curve. When the critical load factor is reached, if a further load increase is applied to the structure, the snap-through phenomenon occurs as the new equilibrium configuration corresponds to very large displacements (more than 4 meters), which imply the structure reversal. 


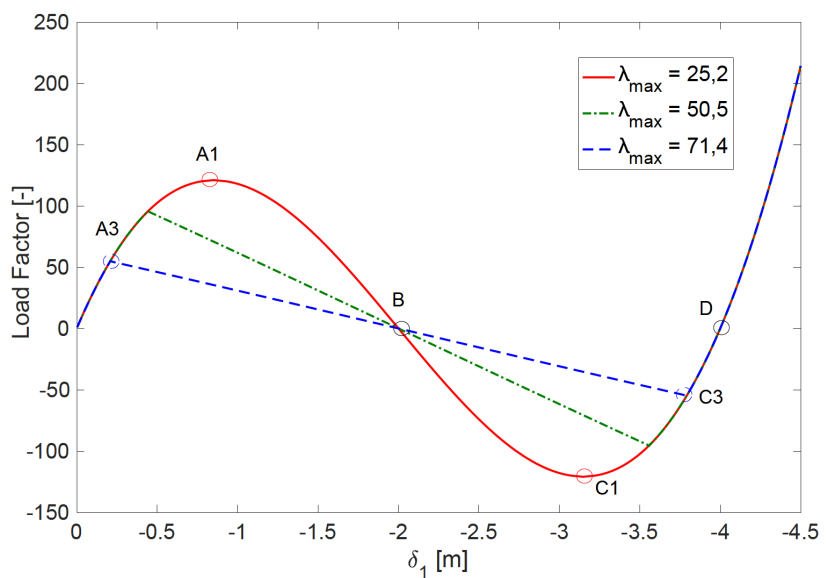

Figure 4: Eight-member structure load-displacement curve

In Figures 5 and 6, some deformed configurations are reported referring to the points shown in Figure 4 for $\lambda_{\max }$ equal to 25.2 (Case 1) and 71.4 (Case 3). As can be noted, the critical condition is associated to different displacement values for the two cases: 0.85 meters for Case 1 (point A1) and 0.21 meters for Case 3 (point A3). Moreover, the unstable branch of the equilibrium path is associated with the Eulerian buckling in Case 3 (A3-B-C3), whereas no Eulerian instabilities occur in Case 1 (A1-B-C1).

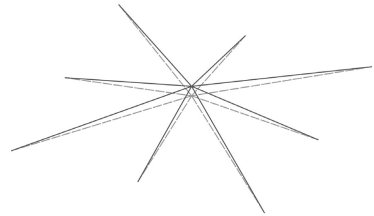

A1

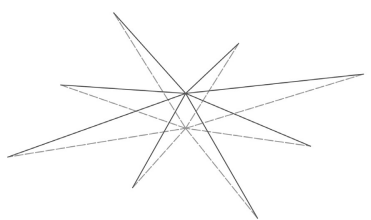

$\mathrm{C} 1$

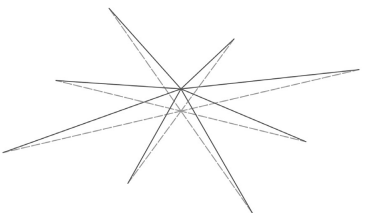

B

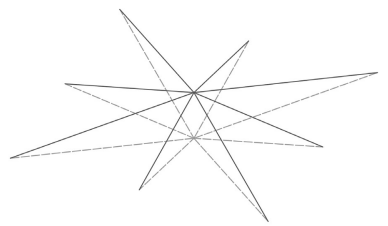

D
Figure 5: Eight-member structure displacements $-\lambda_{\max }=25.2$

\subsection{Four-member lattice structure with one free node}

The second example refers to a structure made up of four constrained nodes and one free node, which is shown in Figure 7. The structure is composed by a simple twomember shallow truss (Figure 7b), stiffened by two hori-

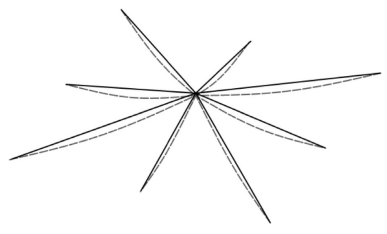

A3

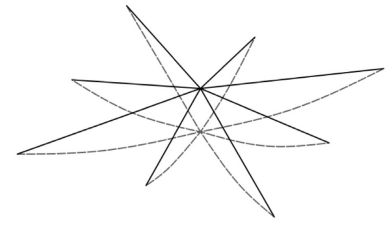

C3
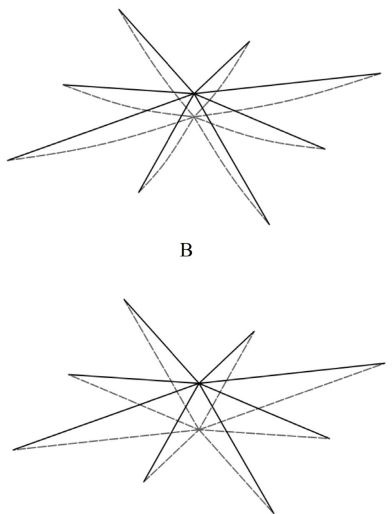

D
Figure 6: Eight-member structure displacements $-\lambda_{\max }=71.4$

zontal transverse bars (Figure 7c). The structure is subject to a downward force applied on the free node, whose initial value is equal to $20 \mathrm{kN}$.
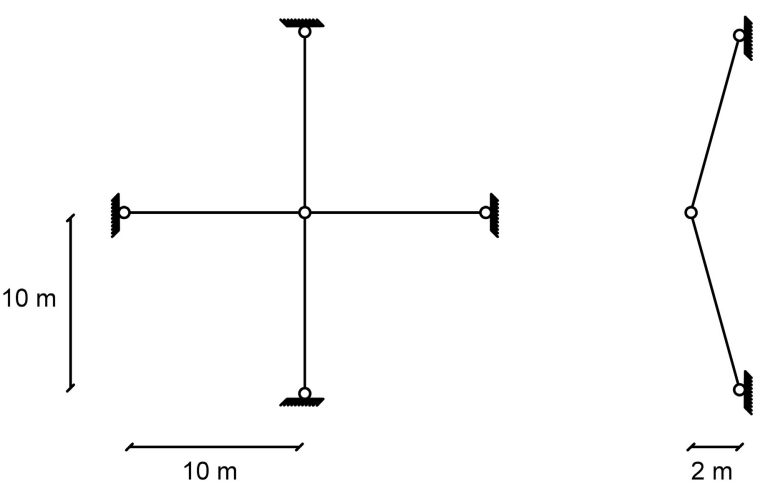

(a)

(b)

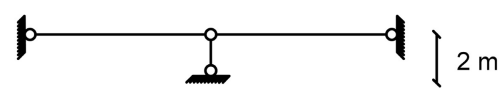

(c)

Figure 7: Geometry of the four-member lattice structure: (a) top view; (b) lateral view; (c) frontal view

In order to study the effect of transverse stiffeners to the structural behavior of the reticular dome, three Cases are considered by varying the cross-sectional area of the horizontal bars $\left(\mathrm{A}_{t}\right)$, whereas the cross-sectional area of the inclined bars (A) is kept constant, as shown in Table 2. The other geometrical and mechanical properties are the same for the four bars.

In Figure 8 the equilibrium paths of the structure are reported for the three cases. As can be noted, by increas- 
Table 2: Four-member lattice structure properties

\begin{tabular}{cccc}
\hline Case & 1 & 2 & 3 \\
\hline $\mathrm{E}[\mathrm{GPa}]$ & 30 & 30 & 30 \\
$\mathrm{~A}\left[\mathrm{~cm}^{2}\right]$ & 100 & 100 & 100 \\
$\mathrm{I}_{\min }\left[\mathrm{cm}^{4}\right]$ & $3.2 \cdot 10^{5}$ & $3.2 \cdot 10^{5}$ & $3.2 \cdot 10^{5}$ \\
$\mathrm{~A}_{t}\left[\mathrm{~cm}^{2}\right]$ & 0 & 25 & 50 \\
$\alpha_{\text {crit }}[-]$ & 45 & 48 & - \\
\hline
\end{tabular}

ing the cross-sectional area of the transverse bars, which are always subject to tensile stress, the unstable branch of the equilibrium path gets smaller and smaller, until it completely disappears in Case 3. Consequently, the snapthrough instability occurs just in Case $1\left(\alpha_{c r i t}=45\right)$ and Case $2\left(\alpha_{c r i t}=48\right)$, whereas no instability phenomena are present in Case 3, which shows just a hardening-stiffening behavior (see Figure 8 and Figure 1a).

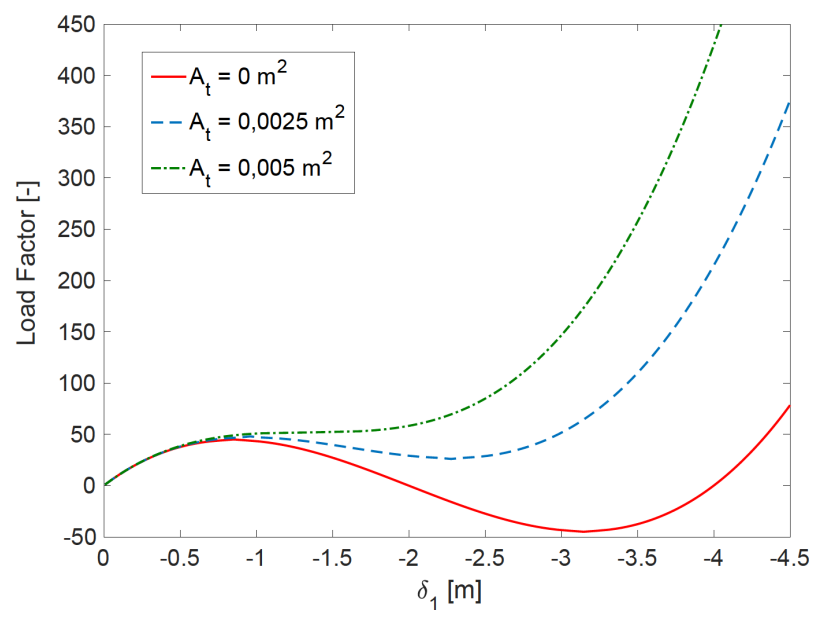

Figure 8: Four-member structure load-displacement curve

\subsection{Sixteen-member lattice structure with five free nodes}

The third example refers to a two-level structure made up of four constrained nodes and five free nodes, as shown in Figure 9. While in the previous examples only one free node was considered, therefore the system reported in Eq. (2) was just made up of one equation in function of one unknown, in the present example five equations depending on five unknowns, i.e. the five vertical displacements of the five free nodes, are considered. The load scheme is identified by a downward vertical force initially equal to
$100 \mathrm{kN}$ applied on the top node. The Young's Modulus of all the bars has been set equal to $30 \mathrm{GPa}$.

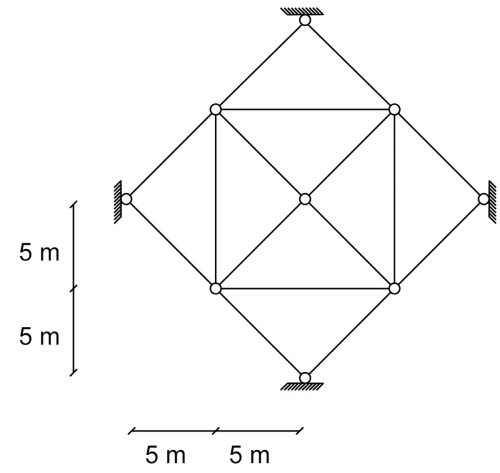

(a)

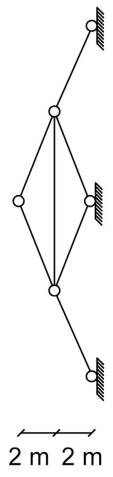

(b)
Figure 9: Geometry of the sixteen-member lattice structure: (a) top view; (b) lateral view

In order to investigate the influence of the Eulerian buckling on the global equilibrium path, two Cases are considered regarding the geometrical properties of the bars: in Case 1 the cross-sectional area and the minimum moment of inertia are equal for all the bars, whereas in Case 2 a lower minimum moment of inertia was considered for the upper bars (i.e. for the bars of the upper level). The geometrical properties used for the analysis are shown in Table 3 (the subscripts down and up indicate the bars belonging to the lower or the upper level, respectively).

Table 3: Sixteen-member lattice structure properties

\begin{tabular}{ccc}
\hline Case & 1 & 2 \\
\hline $\mathrm{A}\left[\mathrm{cm}^{2}\right]$ & 100 & 100 \\
$\mathrm{I}_{\text {min }, \text { down }}\left[\mathrm{cm}^{4}\right]$ & $3.2 \cdot 10^{5}$ & $3.2 \cdot 10^{5}$ \\
$\mathrm{I}_{\text {min, up }}\left[\mathrm{cm}^{4}\right]$ & $3.2 \cdot 10^{5}$ & $0.4 \cdot 10^{5}$ \\
$\lambda_{\text {down }}[-]$ & 13.0 & 13.0 \\
$\lambda_{\text {up }}[-]$ & 13.0 & 36.7 \\
$\alpha_{\text {crit }}[-]$ & 49 & 22 \\
\hline
\end{tabular}

In Figures 10 and 11, the equilibrium paths are reported for Case 1 and 2, respectively. In the left graphs the curves regarding the load factor in function of the top node displacement $\left(\delta_{1}\right)$ are shown, while the right graphs represent the load-displacement curve with respect to the lower nodes $\left(\delta_{2}\right)$. Note that Figure 10a is consistent with the load-deflection curve obtained by Hrinda [17], who analyzed a similar miniaturized structure by using an arc- 

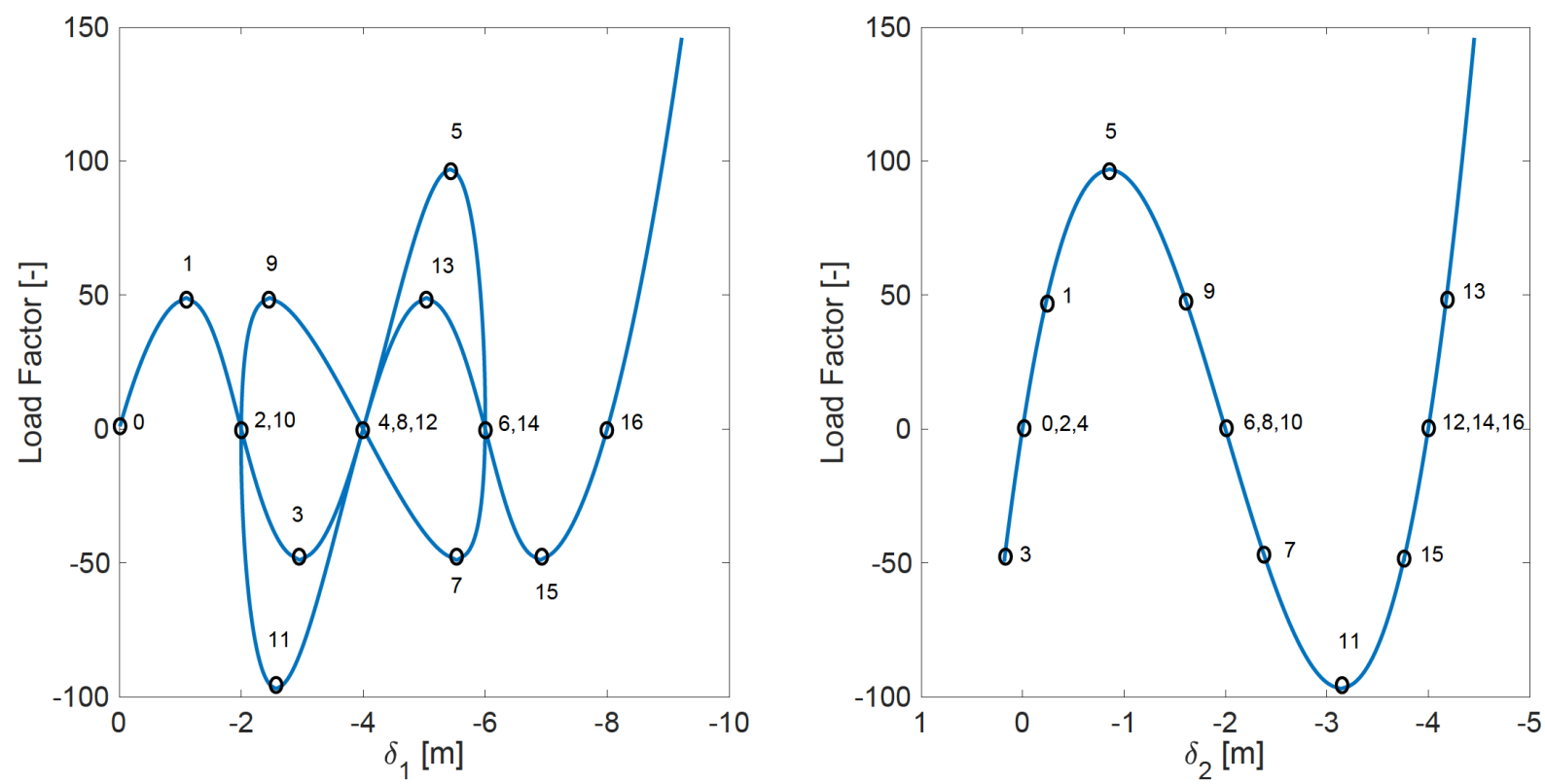

Figure 10: Sixteen-member structure load-displacement curves (Case $1-\lambda_{\text {up }}=13.0$ )
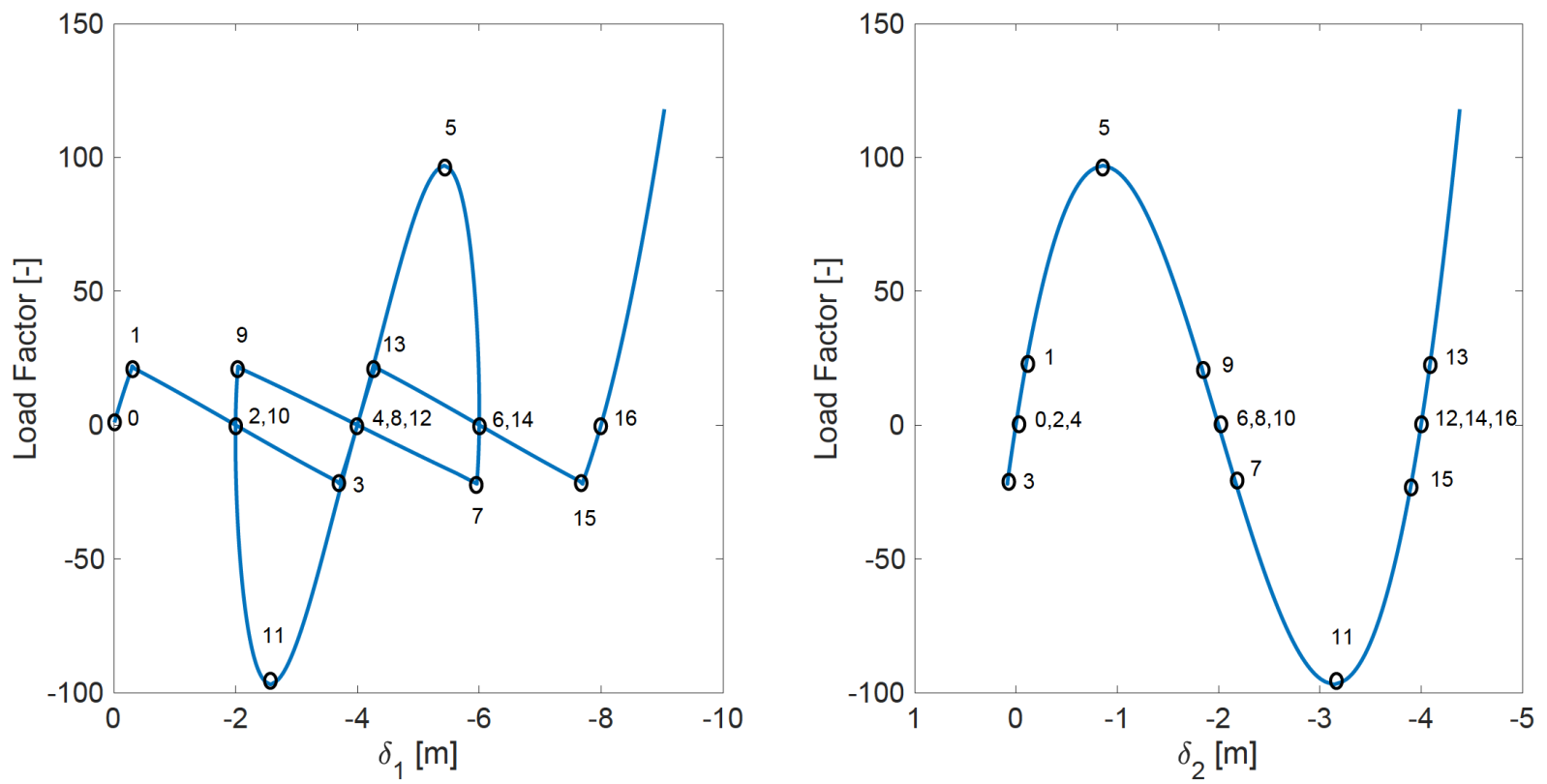

Figure 11: Sixteen-member structure load-displacement curves (Case $2-\lambda_{u p}=36.7$ )

length approach within a Finite Element framework. This indicates that, in this case, the horizontal displacements of the nodes in the lower level (which are not considered in the present formulation) have not a strong influence on the structural response for the selected structure and load scheme.

The following features in the equilibrium paths can be recognized, referring to the portions of the curves identified by the marker points in Figures 10 and 11:
(1) 0-1: an increase in the external load corresponds to an increasing downward displacement in each free node. Marker points 1 identify the critical load factors in each figure: at these points, if the load further increases, the upper part of the structure abruptly changes its configuration, leading to a snap-through-like instability. Note that in Case 2, marker point 1 corresponds to a lower critical load factor $\left(\alpha_{c r i t}=22\right)$ than in Case $1\left(\alpha_{c r i t}=49\right)$, since 
the former is associated with a local Eulerian instability, identified by the bifurcation point within the load-deflection curve. This confirms that, also for more complex structures, the local buckling conditions are able to trigger the snap-through instabilities at lower loading levels;

(2) 1-2-3: in this region, the equilibrium paths imply that the top node keeps moving downward, whereas the lower nodes are progressively going back upward as the load is decreasing. Although both branches represent unstable domains in the load-deflection curves, the branch associated with Case 2 implies the local buckling of the upper members, whereas no Eulerian instabilities are present in Case 1;

(3) 3-4-5: the load starts to increase again, and both the top node and the lower nodes are moving downward. In correspondence to marker points 5 , a second critical load factor can be identified, since at these points, if the load further increases, another abrupt configurational change occurs involving the lower portion of the structure. Note that there are no differences between Case 1 and Case 2 regarding the position of marker point 5 , due to the fact that no Eulerian buckling of the lower part of the structure is found to occur;

(4) 5-6-7: in this region of the load-deflection curves, while the load is decreasing and the lower nodes are moving downward, the top down node initially gets down and then starts to go back upward;

(5) 7-8-9: starting from the previous equilibrium configuration, an increase of the external load corresponds to an increasing upward displacement in each free node. Note that in Case 2, in this region of the equilibrium path, the local buckling of the upper part of the structure occurs, due to the presence of bifurcation points;

(6) 9-10-11: symmetrically to the branch 5-6-7, while the load is decreasing and the lower nodes are moving downward, the top down node initially gets up and then starts to go back downward. Note that after marker point 10 the lower nodes are associated to downward displacements bigger than 2 meters, therefore they are located at a lower level with respect to the edge plane;

(7) 11-12-13: in this sequence, as in 0-1 and 3-4-5, an increase of the external load implies an increasing downward displacement in each free node;

(8) 13-14-15: the top node keeps on moving downward, whereas the lower nodes are progressively going back upward as the load is decreasing, as in 1-2-3. Note that, again, in Case 2 this region of the load- deflection curve is characterized by the Eulerian instability of the upper members;

(9) 15-16-: from this point on, increasing the external load leads to an increasing downward displacement in each free node, as the structure is completely overturned. In correspondence to marker point 16 , the structure is characterized by a configuration which is symmetrical to the initial one with respect to the edge plane. Therefore, from this points on, all the bars are subject to tensile axial forces, a stiffening behavior arises in the equilibrium paths and no Eulerian buckling can occur any longer.

In Figures 12 and 13, the deformed shapes of the structure are reported for the marker points reported in Figures 10 and 11 respectively. In Figure 13 only the marker points associated with Eulerian buckling conditions are represented, since the other ones are identical to those from Case 1. As can be seen, if the complete equilibrium path is followed, the structure undergoes large displace-

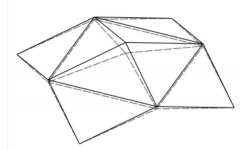

1
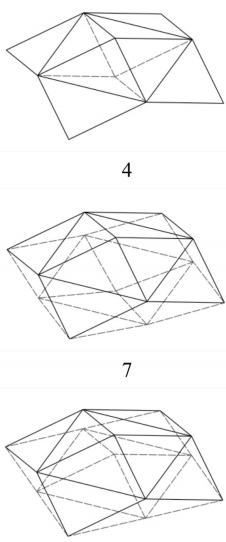

10

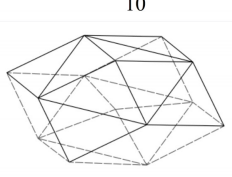

13

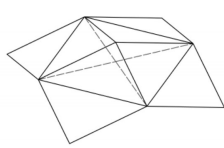

2
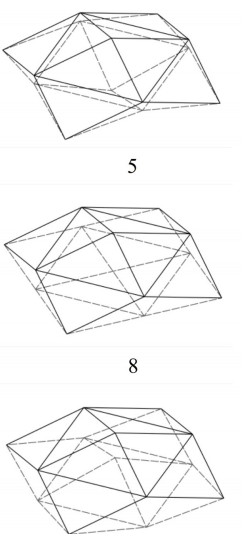

11

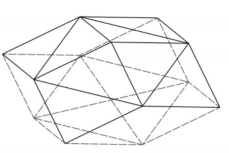

14

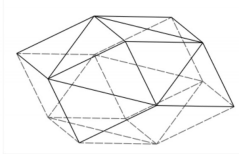

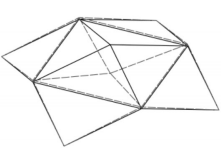

3

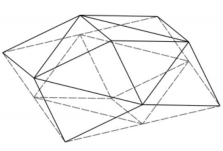

6
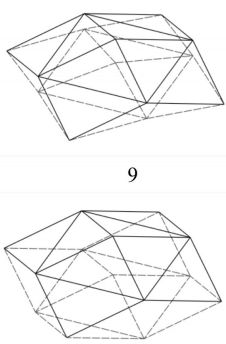

12

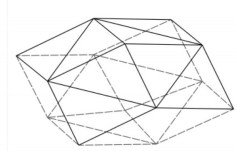

15
Figure 12: Sixteen-member structure displacements (Case $1-\lambda_{\text {up }}=$ 13.0) 


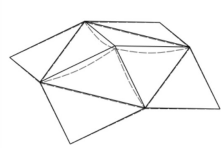

1

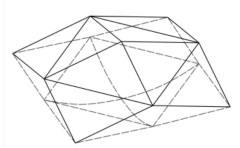

7

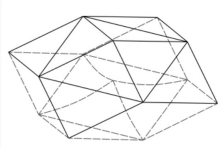

13

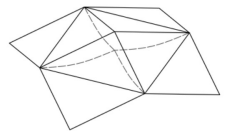

2

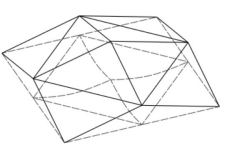

8

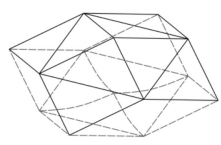

14

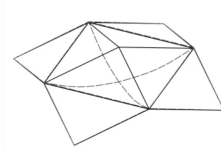

3
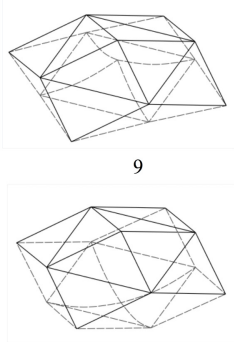

15
Figure 13: Sixteen-member structure displacements (Case $2-\lambda_{\text {up }}=$ 36.7)

ments, which can also lead to the reversal of the structure (e.g. Figure 12 points 11-16, and Figure 13 points 13-15).

The information about the equilibrium path contained in Figures 10 and 11 can be shown in a single representation, putting together the information regarding the load multiplier, the displacement of the top node and the displacements of the lower nodes. This leads to a threedimensional representation of the load-deflection curves, which is shown in the left graphs of Figures 14 and 15 for Case 1 and Case 2, respectively. By observing the threedimensional representations, it is interesting to note how the equilibrium paths imply continuous lines without self- intersections, i.e. all the points lying on the curve are characterized by a single specific combination of coordinates (displacement of the top node, displacement of the lower nodes, load multiplier). The graphs shown in Figures 10 and 11 can then be seen as the projection on vertical planes (load multiplier vs displacement of the top node, load multiplier vs displacement of the lower nodes) of the threedimensional curves in Figures 14 and 15.

In the right graphs of Figures 14 and 15, the top projections of the three-dimensional curves are also reported, showing the pathway followed by the nodes during the loading process ( $\delta_{1}$ vs $\delta_{2}$ ). As can be seen, the pathway is rather complex involving various nonlinear branches. When the loading process comes to the structural configuration associated to the marker point 6 , the displacement of the top node is supposed to decrease to guarantee the equilibrium. However, if the loading process is controlled by increasing the displacement of the top node, then the branch from marker point 6 to marker point 14 becomes virtual, and the structure suddenly snaps to the configuration associated to marker point 14 (see the black dotted arrow in the graphs). This new configuration is characterized by the same displacement of the top node (6 meters), while the lower nodes experience an abrupt increase of their displacements (from 2 meters up to 4 meters).

In the same way, if the loading process is controlled by continuously increasing the downward force, the equilibrium paths are not covered entirely and the resulting load-deflection curves can be divided into three main
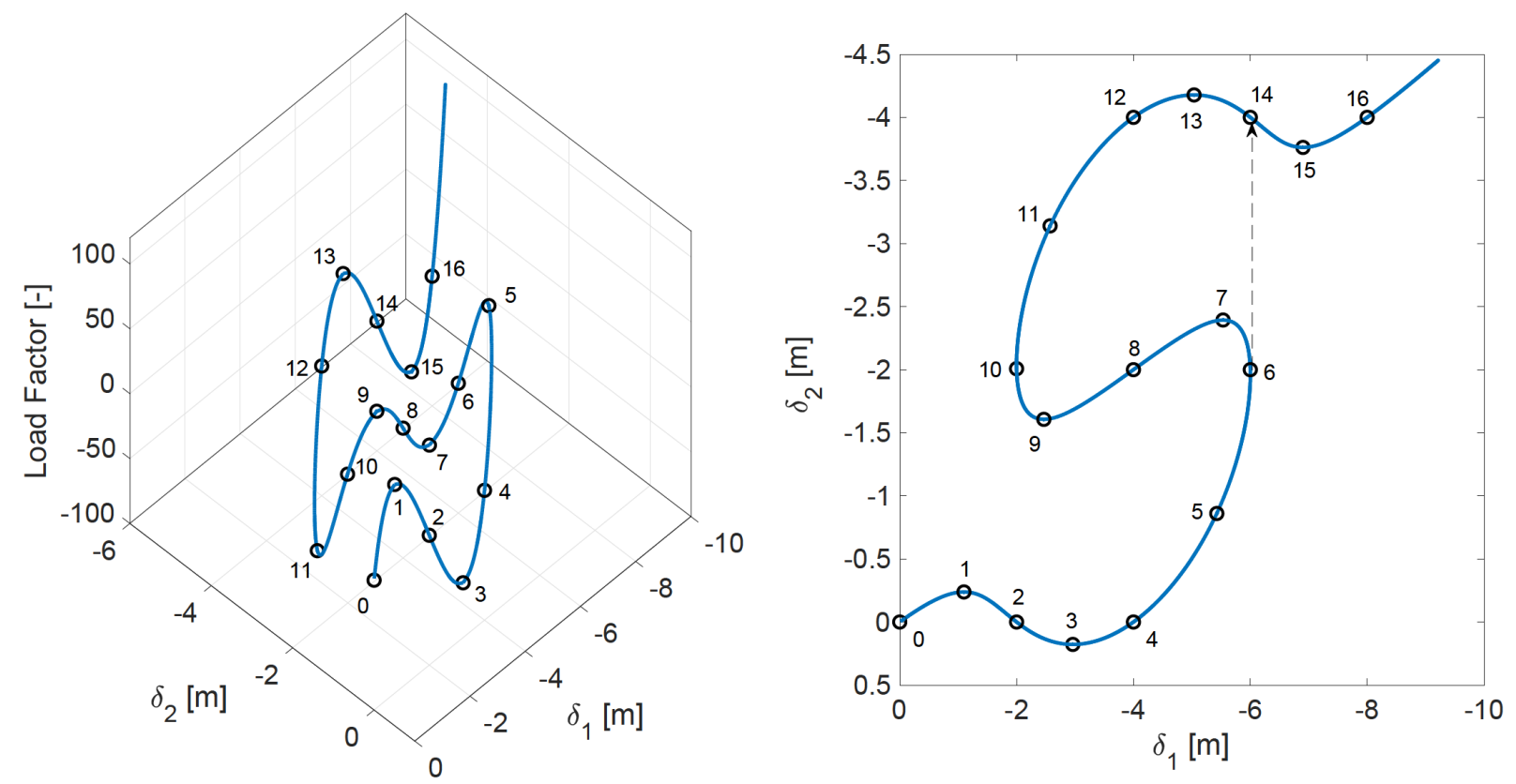

Figure 14: Sixteen-member structure load-displacement curve (Case $\left.1-\lambda_{u p}=13.0\right)$ : three-dimensional view (left) and top projection (right) 

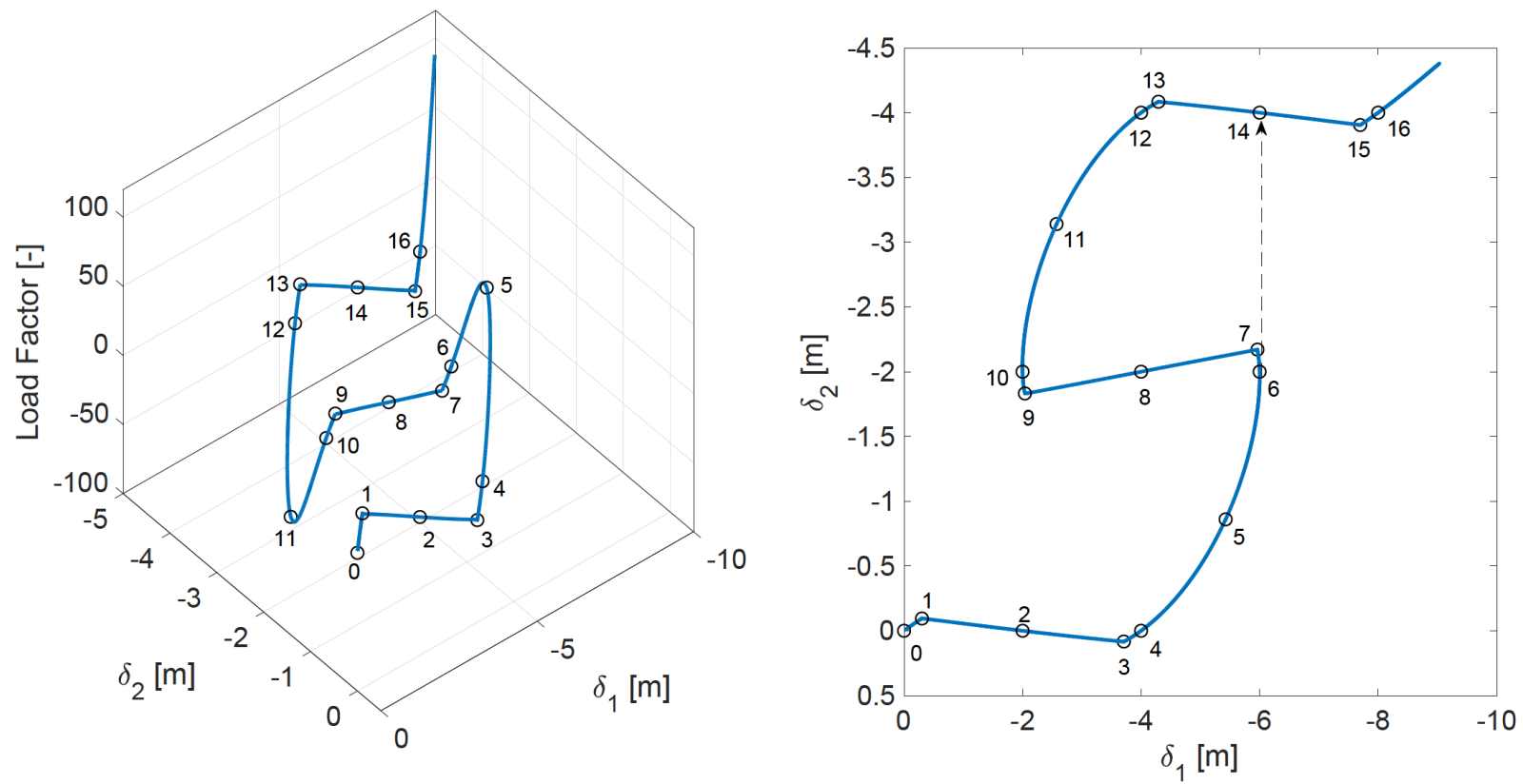

Figure 15: Sixteen-member structure load-displacement curves (Case $2-\lambda_{u p}=36.7$ ): three-dimensional view (left) and top projection (right)
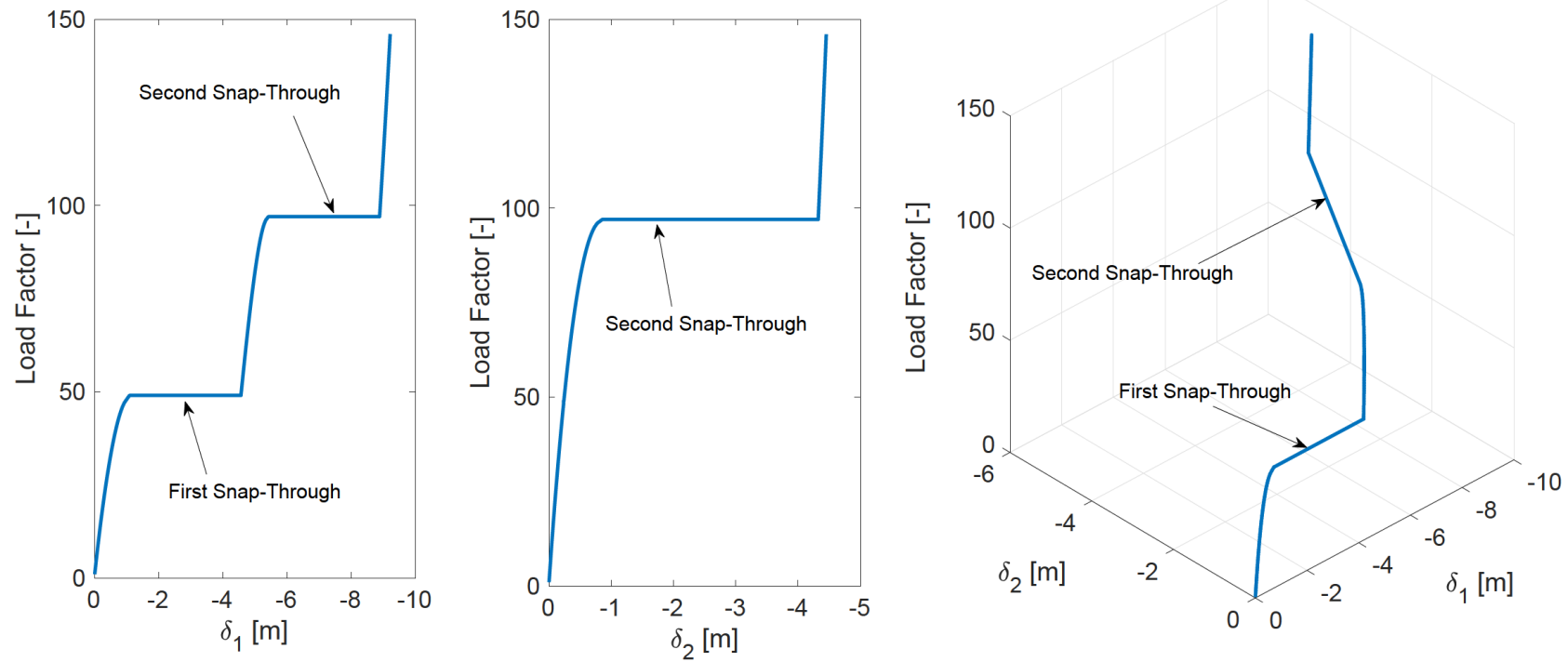

Figure 16: Sixteen-member structure load-displacement curves for increasing load (Case 1)

sequences, involving two subsequent snap-through phenomena (Figures 16 and 17):

1. Initially, the branch $0-1$ is covered. When marker point 1 is reached, if the load still increases, the new equilibrium configuration is found on the branch 45 , at the same critical load factor correspondent to marker point 1 (Figures 16 and 17). As can be seen from the equilibrium paths referring to the top and lower nodes (Figures 10 and 11), this snap-through phenomenon involves only the top node, as it exhibit a large downward displacement, higher than 4 meters. Contrariwise, it does not involve the lower nodes, since they remain in the same position they occupied in correspondence of marker point 1 ;

2. The load being increased again, the equilibrium branch is travelled towards marker point 5 . When this point is reached, if the load still increases, a new equilibrium configuration is found on the last 

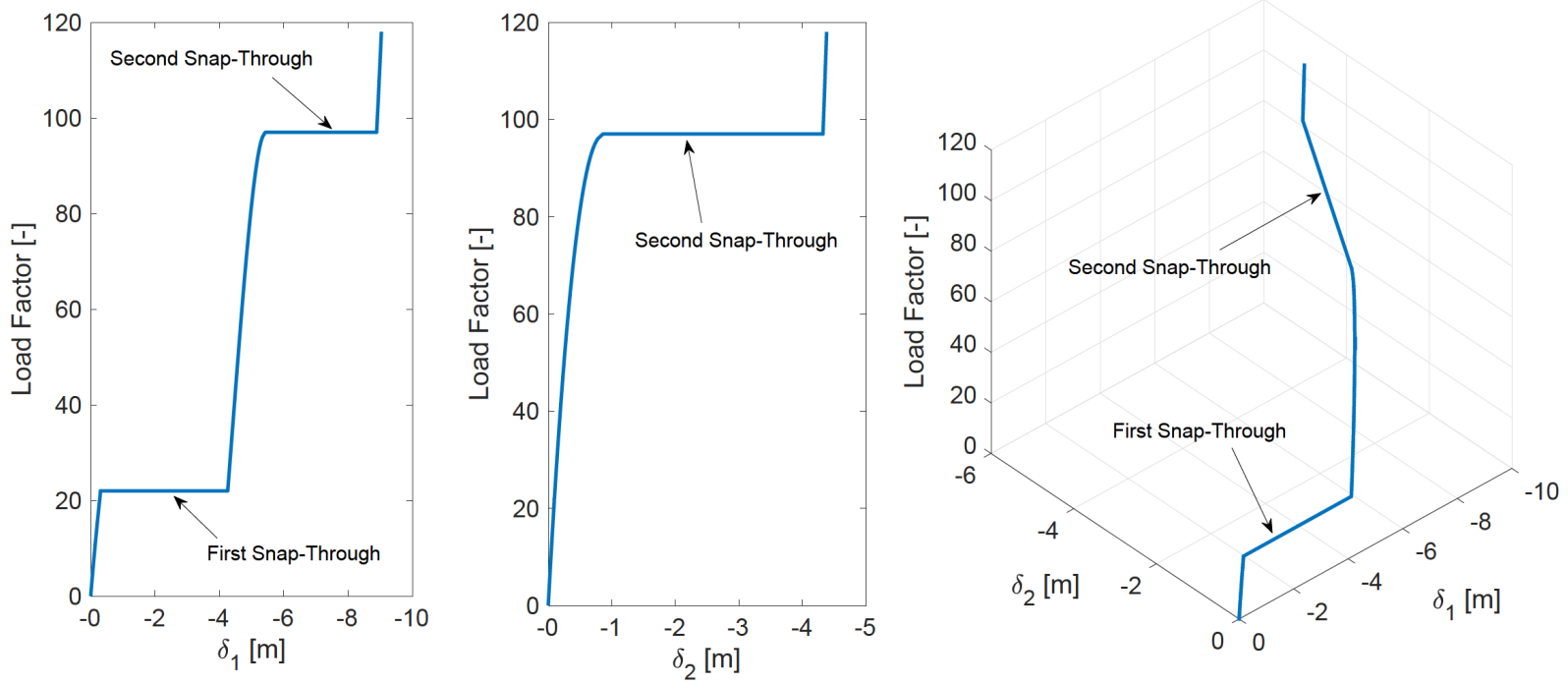

Figure 17: Sixteen-member structure load-displacement curves for increasing load (Case 2)
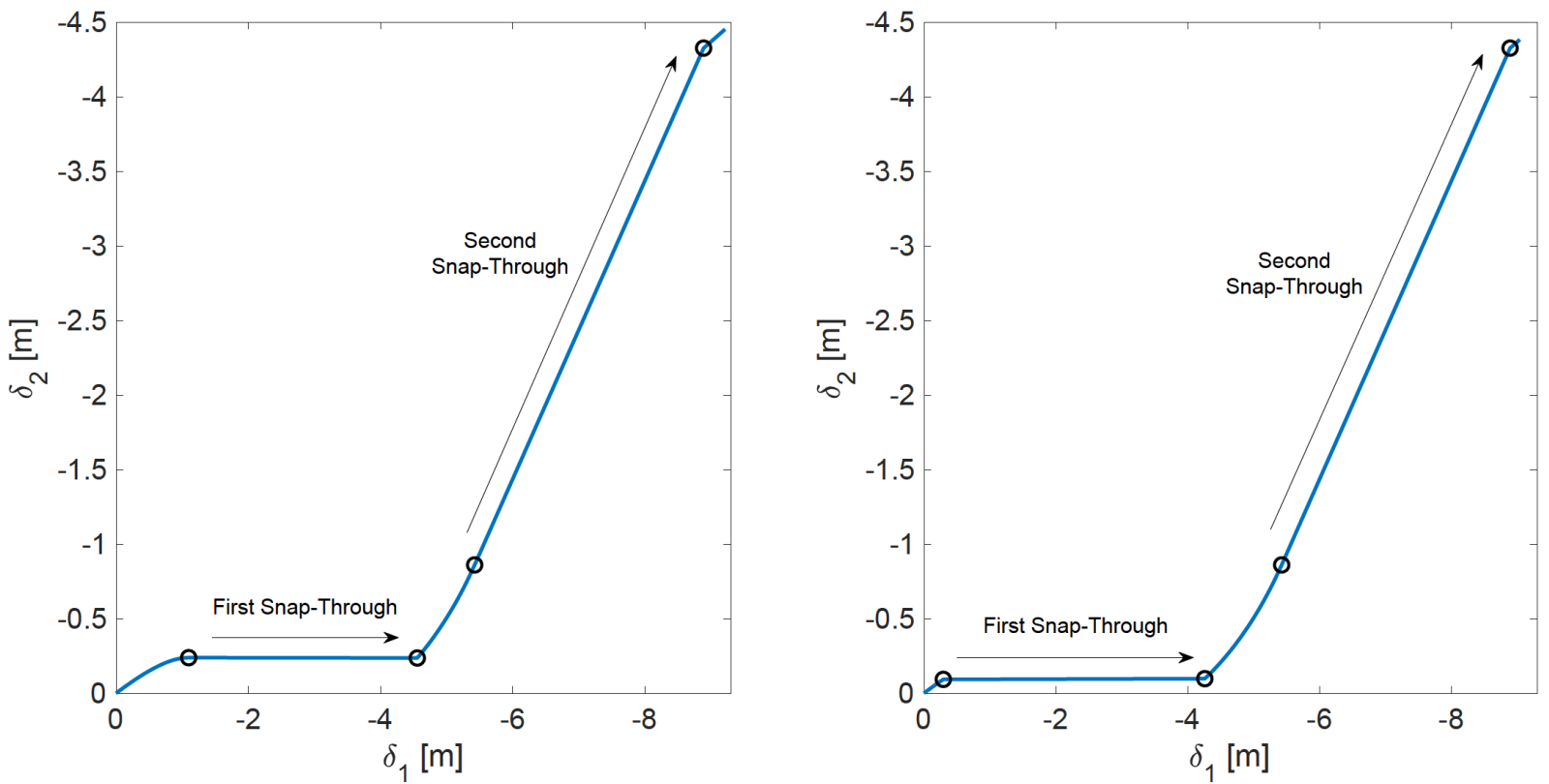

Figure 18: $\delta_{1}$ vs $\delta_{2}$ curves for increasing load: Case 1 (left graph) and Case 2 (right graph)

branch after marker point 16 , at the same critical load factor correspondent to marker point 5 (Figures 16 and 17). As can be seen from the equilibrium paths (Figures 10 and 11), in this case the snapthrough involves both the top node, which exhibits a large downward displacement (higher than 8 meters), and the lower nodes, which exhibit a downward displacement (higher than 4 meters). At this point the structure is completely overturned;

3. From this moment on, the structure is only subject to tensile axial forces and the equilibrium path of the structure assumes a stiffening behavior, since further loading increases cause increasingly smaller displacements both in the top and in the lower nodes (Figures 16 and 17).

In Figure 18 the displacement pathway which is followed by the top and lower nodes is shown, for Case 1 (left graph) and Case 2 (right graph). Note that these graphs are the projections of the three-dimensional curves shown in Figures 16 and 17, respectively. The first snap-through clearly implies a 'snapping' phenomenon involving only the top nodes, whereas the second snap-through involves 
mainly the lower nodes. The top node undergoes another significant displacement which is just caused by the sudden downward displacements of the lower portion of the structure. It is clear that, both in Case 1 and Case 2, these snap-through instabilities can be accompanied by significant dynamic effects. If these effects are strong enough to compromise the resistance of the members, a sudden failure of the structure can occur and the equilibrium curves are interrupted.

The difference between Case 1 (Figure 16) and Case 2 (Figure 17) is that, while in Case 1 no Eulerian buckling occurs, in Case 2 the upper bars undergo local Eulerian instability. This phenomenon lowers the critical load factor associated to the first snap-through phenomenon, which is related to the upper part of the structure; contrariwise, since the second snap-through is only related to the lower part of the structure, there are no differences concerning the value of the second critical load factor $\left(\alpha_{c r i t, 2}=97\right)$.

\section{Conclusions}

In designing thin, slender or long-span structures, linear analysis could be not enough to fully understand the structural behavior. As a matter of fact, nonlinearities can occur both considering material and geometrical parameters, and can cause important consequences in the design choice. This is especially true for lattice domes, whose designing criteria can be influenced not only by material resistance, but also by snap-through phenomena and local Eulerian buckling. All these aspects are usually investigated using Finite Element Method simulations.

In this paper, we made use of a developed semianalytical formulation which is meant to study the geometrically nonlinear behavior of lattice structures subject to vertical load. The approach is based on writing the equilibrium equations in the deformed configuration of the structure. Although a linear constitutive law is considered regarding the material, the Eulerian critical axial load is assumed to represent the maximum compressive load which the bars can bear: in this way, it was possible to perform a geometric nonlinear analysis coupled with the local Eulerian buckling instabilities. Due to the fact that the analytical functions involved a system of nonlinear equations, dedicated MATLAB ${ }^{\circledR}$ functions were used in order to solve the structural problem and to follow the whole equilibrium paths, by means of a dedicated mixed load-displacement control scheme.

Via this approach, the geometrically nonlinear behavior of some space lattice structures was investigated, e.g. showing the influence of some geometrical parameters on the equilibrium paths, such as the bar slenderness and the cross-sectional area of transverse stiffeners. A complex load-deflection curve was also investigated regarding a sixteen-member lattice structure and several regions of the equilibrium path were identified depending on the evolution of the load factor and the nodes displacements. Also in this case, the influence of the bar slenderness on the modification of the branches of the load-deflection curve was examined.

The last example, i.e. the sixteen-member dome, highlighted the complexity hidden behind the nonlinear behavior of spatial lattice structures. The geometrical nonlinearities cause the load-deflection curves to deviate from the linearity and induce post-critical branches which can be associated to both stable and unstable conditions, depending on the value of the load and corresponding structural configuration. Various snap-through and snap-back branches can occur in the unstable regions, which depend not only on the specific geometrical properties of the structure but also on the loading scheme. Although these branches may be not of interest for designing purposes, since these structures are designed to work under much lower loads than the critical ones, they can provide important information on their intrinsic structural stability. The knowledge of the whole load-deflection curve, with the information on the possible post-critical behaviors, can in fact claim attention on the likeliness for the structure to experience the various instability phenomena.

Tracing such complex curves through experimental tests, e.g. in laboratory, would be unachievable, due to the difficulty to manage many degrees of freedom and because of the presence of imperfections and material nonlinearities, which can modify the structural response. For this reason, Finite Element simulations and ad-hoc analytical formulations, like the one used here, are useful to investigate the occurrence of such post-critical behaviors. The presented semi-analytical methodology has then been developed as a simple yet powerful tool to shed light on these critical phenomena. At this stage, it does not take into account material nonlinearities and geometrical imperfections, which are known to play a key role in modifying both the critical load multiplier and the equilibrium path. In future contributions, this formulation will be enriched by considering also nonlinear constitutive laws for the bars included within the three-dimensional domes. The presence of horizontal forces and horizontal displacements will be taken into account as well, in order to consider more complex loading schemes. 
Conflict of Interest: The authors declare no conflict of interest regarding the publication of this paper.

\section{References}

[1] Fu F., Progressive collapse design and analysis of space structures. From: Structural analysis and design to prevent disproportionate collapse, CRC Press, 2016.

[2] Thompson J. M. T., Basic principles in the general theory of elastic stability. J Mech Phys Solids, 1963, 11, 13-20.

[3] Von Karman T., Tsien H. S., The buckling of spherical shells by external pressure, J Aeron Sciences, 1939, 7, 43-50.

[4] Von Karman T., Dunn L. G., Tsien H. S., The influence of curvature on the buckling characteristics of structures, J Aeron Sciences, 1940, 7, 276-289.

[5] Von Karman T., Tsien H. S., The buckling of thin cylindrical shells under axial compression, J Aeron Sciences, 1941, 8, 898-907.

[6] Michielsen H. F., The behavior of thin cylindrical shells after buckling under axial compression, J Aeron Sciences, 1948, 15, 738-744.

[7] Bushnell D., Buckling of shells - Pitfall for designers, AIAA J, 1981, 19, 1183-1226.

[8] Carpinteri A., Cusp catastrophe interpretation of fracture instability. J Mech Phys Solids, 1989, 37, 567-582.

[9] Carpinteri A., Paggi M., Zavarise G., Cusp-catastrophe interpretation of the stick-slip behaviour of rough surfaces, CMES-Comp Model Eng, 2009, 53, 303-325.

[10] Gioncu V., Buckling of Reticulated Shells: State-of-the-Art, Int J Space Struct, 1995, 10, 1-46.

[11] Cai J., Xu Y., Feng J., Zhang J., Nonlinear stability of a single-layer hybrid grid shells, J Civ Eng Manag, 2012, 18, 752-760.

[12] Fan F., Yan J., Cao Z., Stability of reticulated shells considering member buckling, J Constr Steel Res, 2012, 77, 32-42.
[13] Lefevre B., Douthe C., Baverel O., Buckling of elastic gridshells, IASS J, 2015, 56, 153-171.

[14] Gioncu V., General theory of coupled instability, Thin Wall Struct, 1994, 19, 81-127.

[15] Gioncu V., Consistent simplified theory for elastic coupled instability, Thin Wall Struct, 1994, 19, 147-159.

[16] Bazzucchi F., Manuello A., Carpinteri A., Interaction between snap-through and Eulerian instability in shallow structures, Int J Non Linear Mech, 2017, 88, 11-20.

[17] Hrinda G. A., Snap-Through Instability Patterns in Truss Structures, $51^{\text {st }}$ AIAA/ASME/ASCE/AHS/ASC Structures, Structural Dynamics, and Materials Conference, Orlando, Florida, 2010.

[18] Pecknold D. A., Ghaboussi J., Healey T. J., Snap-Through and Bifurcation in a Simple Structure, J Eng Mech, 1985, 111, 909922.

[19] Morris N. F., Effect of member snap on space truss collapse, J Eng Mech, 1993, 119, 870-886.

[20] Bazzucchi F., Manuello A., Carpinteri A., Instability load evaluation of shallow imperfection-sensitive structures by form and interaction parameters, Eur J Mech A Solids, 2017, 66, 201-211.

[21] Crisfield M. A., The automatic non-linear analysis of stiffened plates and shallow shells using finite elements, P I Civil Eng, 1980, 69, 891-909.

[22] Crisfield M. A., A fast incremental/iterative solution procedure that handles "snap-through", Comput Struct, 1983, 13, 55-62.

[23] Lam W. F., Morkley C. T., Arc-length method for passing limit points in structural calculation, J Struct Eng, 1992, 118, 169-185.

[24] Bruns T. E., Sigmund O., Tortorelli D. A., Numerical methods for the topology optimization of structures that exhibit snapthrough, Int J Numer Meth Eng, 2002, 55, 1215-1237.

[25] Liu N., Plucinsky P., Jeffers A. E., Combining load-controlled and displacement-controlled algorithms to model thermalmechanical snap-through instabilities in structures, J Eng Mech, 2017, 143, 04017051. 